\title{
Traumatic Closed Floating Kneecap: Clinical Case about Traumatic Bipolar Tendon Rupture of Extensor Knee System
}

\author{
Koné Sam*, Pemboura Dom, Eric Dogba, Akué Koffi, Abdoulaye Bana, Serge Agoh \\ Service de traumatologie-Orthopédie, CHU de Cocody-Abidjan, Abidjan, Cote D’Ivoire \\ Email: ^k.samba06@gmail.com
}

How to cite this paper: Sam, K., Dom, P., Dogba, E., Koffi, A., Bana, A. and Agoh, S. (2019) Traumatic Closed Floating Kneecap: Clinical Case about Traumatic Bipolar Tendon Rupture of Extensor Knee System. Open Journal of Orthopedics, 9, 159-164. https://doi.org/10.4236/ojo.2019.98017

Received: June 29, 2019

Accepted: August 6, 2019

Published: August 9, 2019

Copyright $\odot 2019$ by author(s) and Scientific Research Publishing Inc. This work is licensed under the Creative Commons Attribution International License (CC BY 4.0).

http://creativecommons.org/licenses/by/4.0/

\begin{abstract}
The simultaneous traumatic rupture of the patellar tendon and the quadricipital tendon is a rare lesion entity. We report the case of a bipolar rupture of the extensor knee system in a young adult following sports traumatism. Magnetic Resonance Imaging allowed the assessment of associated lesions. The management of this "floating" kneecap was surgical associated with rehabilitation. A functional assessment at 02 years of follow-up was performed. The functional prognosis is related on the one hand to the difficulties of positioning of the patella and on the other hand to the associated lesions (meniscus-ligamentous knee injuries).
\end{abstract}

\section{Keywords}

Rupture, Bipolar, Extensor Knee System

\section{Introduction}

Extensor knee system (EKS) traumatic rupture is fairly common lesions. It can be three lesional entities (patella fractures, quadriceps tendon rupture, patellar tendon rupture) which are frequently isolated [1].

These lesions are often of traumatic origin. Sometimes they occur on preexisting lesions or on particular ground (elderly, renal failure) outside any traumatic context [2] [3].

Rigorous physical examination and radiological investigations are paramount in order to make an accurate diagnosis and search for associated lesions. Treatment of these tendon ruptures is surgical with various techniques.

Bipolar ruptures of EKS are rare and raise some therapeutic difficulties. The functional prognosis is related on the one hand to the difficulties of positioning 
of the kneecap, and on the second hand related to the associated lesions (meniscus-ligamentous knee injuries).

The authors report the case of traumatic closed floating kneecap (traumatic bipolar tendon rupture of knee extensor system) in a young adult resulting from sporting trauma. The aim of this work was to highlight this rare clinical entity through a discussion of lesional mechanism and therapeutic modalities and prognosis.

\section{Case Report}

It was about a sporting man of 33 years, without any past medical history; who was received in the emergency room for severe left knee pain with absolute functional impotence following sporting traumatism (basketball).

The physical examination revealed a large painful of left knee, depressions above and under patellar. Radiologic investigations (radiographs, Magnetic Resonance imaging revealed) traumatic rupture of the patellar and quadricipital tendons associated with anterior cruciate and collateral ligaments rupture (Figure 1, Figure 2).

A surgical indication was asked. Ligament testing in the operating room preoperatively noted positive Lachman and frontal and lateral medial laxity. Surgical approach was median (Figure 3). In each operation we carried an assessment lesion: rupture of quadricipital and patellar tendon; rupture of the anterior cruciate ligament.

We started repair with the patellar' tendon, then the quadriceps' tendon. Quadriceps' tendon repair was performed using the Krackow [4] technique. That of the patellar' tendon was done by direct suture and protect him by metal frame (Figure 3). A knee plaster has been put in place for a period of 6 weeks. The postoperative radio showed a high kneecap (Figure 4).

After 6 weeks a passive rehabilitation had been undertaken for 6 weeks. Active physiotherapy had been authorized from the 3 rd post-operative month. The patient was regularly followed and at 9 months of recoil, he did not present any functional complaint, the ligamentous testing (Lachman) delayed hard stop. The mobility of the knee was: extension was normal, the patient had a flexion at $115^{\circ}$ and a flexum residual at $15^{\circ}$.

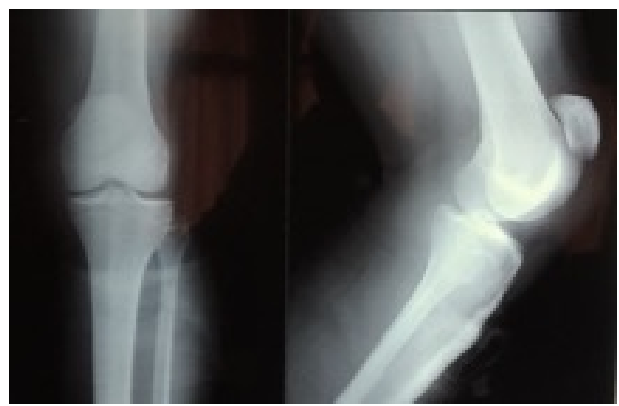

Figure 1. Radiographs (Antero-posterior and Medio-lateral) of left knee showing extensor knee system rupture with high kneecap. 


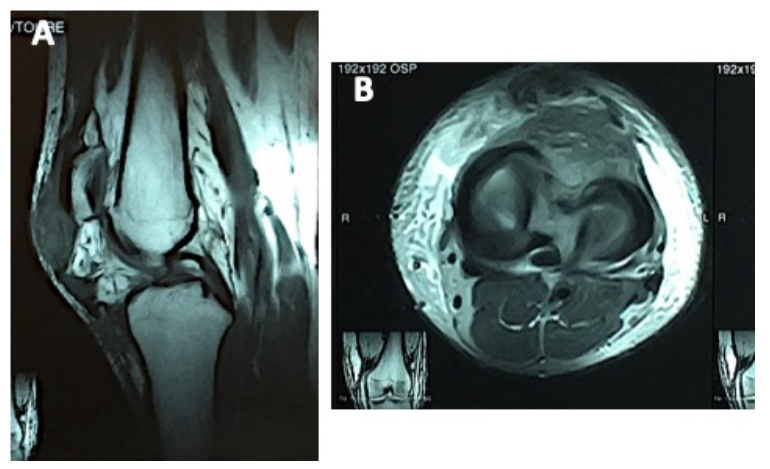

Figure 2. MRI of left knee showing rupture of the patellar and quadricipital tendons associated with anterior cruciate and collateral ligaments rupture. (A) Coronal incidence; (B) Axial incidence.
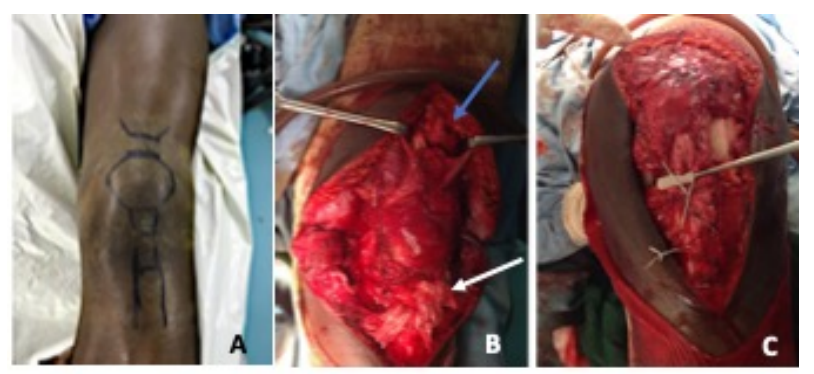

Figure 3. (A) Preoperative clinical aspect; (B) Operative aspect of floating kneecap; rupture quadricipital tendons (blue arrow); rupture of patellar tendon (white arrow); (C) Operative aspect after direct suture and protection by metal frame.
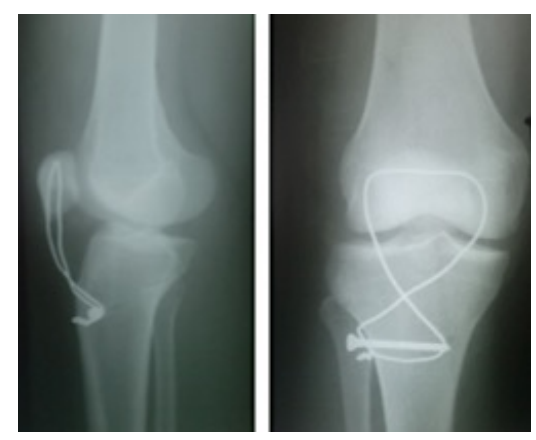

Figure 4. Radiographs (Medio-lateral and Antero-posterior) of left knee showing osteosynthesis by metal frame press a screw, and high kneecap.

We offered him a reconstruction of the anterior cruciate ligament but, the patient refused. At the 2-year follow-up he complains of anterior episodic pain requiring him to convert to another sporting activity (sport in room). In terms of mobility, residual anterior instability persisted.

\section{Discussion}

The purpose of postponing of this unusual clinical case following sport trauma in a young adult was to discuss the injury mechanism and highlight the therapeutic difficulties. "Floating kneecap" is an exceptional lesion. No case has been 
reported so far in sub-Saharan Africa.

Generally, ruptures of the EKS most often occur during a high-energy trauma, as was the case with our patient and those reported in the literature [5]. However, it can spontaneously occur. These are generally attributed to chronic corticosteroids use or systemic diseases [2] [6].

In all cases of mechanical factors (repetitive strain injury), local factors (past history of knee surgery) are considered in the pathogenesis of these ruptures. Several hypotheses are described concerning traumatic tendon rupture mechanism of the EKS [5] [7]. An indirect trauma on a bent knee involving a sudden contraction of the quadriceps on a slightly bent knee or a direct shock on the knee bent at $90^{\circ}$.

We believe that these mechanisms are involved in the occurrence of a floating kneecap. In any cases, it seems difficult to describe chronology of this ipsilateral rupture except hypotheses. Indeed, no objective argument could predict which of the tendons broke first (quadriceps' or patellar' tendon).

The clinical diagnosis was obvious in our patient; however, unknown or intraoperative forms of discovery are reported in the literature [3] [7]. Magnetic resonance imaging remains the primary examination in dubious, unknown or ancient forms.

This one confirms the rupture, appreciates the state of the residual tendon stump, and looks for associated lesions. Associated lesions (meniscus, fracture) with a floating kneecap were sometimes reported [8] [9].

However, associated damage of the anterior cruciate ligament and collateral ligament has not yet been described. This is another peculiarity of our clinical case.

Probably because of the rarity of this lesion, no consensus on surgical technique has yet been reached; All recommend surgical treatment, based on direct U-shaped tendon suture or re-insertion into bone trench supported by trans-osseous points (Krackow technique) [4].

Some recommend early repair (before 3 weeks) of tendons (patellar and quadricipital); beyond 6 weeks, the difficulties in restoring the length of the damaged tendon and therefore the repositioning (centering) of the kneecap increase, because of the existence of adhesions, fibrosis associated with a retraction of the quadriceps and or residual tendon stump.

For this patient we combined the Krackow technique (quadricipital tendon) and direct suture (patella tendon). Patellar' tendon suture was protected by a metal frame (to allow early rehabilitation).

The floating kneecap nature exposes it to failure of repositioning the patella, resulting in secondary femoral-patellar conflict. Indeed, our patient had sequella pain related to a high patella.

In presence of associated lesions such as meniscus or ligamentous injuries the decision to treat them all at the same time is a school matter. The choice of the moment of repairing all lesions is crucial [8]. According to the literature [8] [9] in case of associated ACL injury, the ACL reconstruction is certainly essential 
but cannot be combined with the others because of the risk of arthrofibrosis. This reconstruction should be done on a cold knee away from local inflammatory phenomena. Given our daily practice realities we suggest that initial lesions and associated injury (ACL, collateral ligamentous) be treated independently.

In our patient we delayed the repair of the ACL anterior cruciate ligament which could not take place in front of the patient's refusal. Nevertheless, he was satisfied that he had achieved acceptable knee mobility despite sequelae observed (residual anterior instability and episodic pain).

The functional prognosis of this lesion is related to difficulties of positioning the patella and to associated lesions.

\section{Conclusion}

The floating kneecap is an extremely unusual injury that requires an accurate injury assessment. The MRI provides the practitioner with the information needed to plan and select the most appropriate treatment. Difficulty in repositioning the patella and associated lesions make the functional severity prognosis.

\section{Consent}

The patient and the families were informed that data from the case would be submitted for publication and provided their consent accordingly.

\section{Author's Contributions}

All the authors contributed of the writing of this manuscript and have read and approved the final version.

\section{Conflicts of Interest}

The authors declare that they have no competing interests.

\section{References}

[1] Selmi, T., Neyret, P., Rongieras, F. and Caton, J. (1999) Rupturesde l'appareil extenseur du genou et fractures de rotule. EMC Techch 44-730.

[2] Lu, M., Johar, S., Veenema, K. and Goldblatt, J. (2012) Patellar Tendon Rupture with Underlying Systemic Lupus Erythematosus: A Case Report. Journal of Emergency Medicine, 43, e35-e38. https://doi.org/10.1016/j.jemermed.2009.08.054

[3] Caldas, M.T.L., Barbara, G.H.S. and Bárbara, M.B.F. (2013) Simultaneous Bilateral Rupture of the Patellar Ligament in Chronic Renal Patient. A Case Report. Revista Brasileira de Ortopedia (English Edition), 48, 455-459. https://doi.org/10.1016/j.rboe.2012.08.011

[4] Krackow, K., Thomas, S.C. and Jones, L.C. (1996) A New Stitch for Ligament-Tendon Fixation: Brief Note. The Journal of Bone and Joint Surgery. American Volume, 68, 764-766. https://doi.org/10.2106/00004623-198668050-00020

[5] Song, F., et al. (2009) Traumatic Rupture of the Ipsilateral Quadriceps and Patellar Tendons: A “Floating Patella”. University of Pennsylvania Orthopaedic Journal, 19.

[6] Lombardi, L.J., Cleri, D.J. and Epstein, E. (1995) Bilateral Spontaneous Quadriceps 
Tendon Rupture in a Patient with Renal Failure. Orthopedics, 18, 187-191.

[7] Siwek, C.W. and Rao, J.P. (1981) Ruptures of the Extensor Mechanism of the Knee Joint. The Journal of Bone and Joint Surgery. American Volume, 63, 932-937. https://doi.org/10.2106/00004623-198163060-00010

[8] Achkoun, A., et al. (2016) Rupture simultanée du ligament croisé antérieur et du ligament patellaire: À propos d'un cas. Pan African Medical Journal, 23, 20. https://doi.org/10.11604/pamj.2016.23.20.8621

[9] Woo, S.L.Y., Kanamori, A., Zeminski, J., et al. (2002) The Effectiveness of Reconstruction of the Anterior Cruciate Ligament with Hamstrings and Patellar Tendon. The Journal of Bone and Joint Surgery. American Volume, 84, 907-914. https://doi.org/10.2106/00004623-200206000-00003 